\title{
Replantation of hand after transmetacarpal amputation
}

\begin{abstract}
Background: The first hand replantation was completed in 1962 by Malt and Mckhannin in Boston, since then, multiple case-reports with a success rate of $80-90 \%$ have been published. The Ischemia time and trauma mechanism are the main factors that influence the rate of success directly, however, the treatment must be individualized.
\end{abstract}

Case Report: A 32 years-old male with transmetacarpal amputation at flexor zone II of the left hand after traumatic amputation with a wood saw arrived to emergency room. The Patient was evaluated by the surgical team and concluded the Patient was a good candidate for a hand Replantation mainly because of the short time since the injury and the clean cut aspect. The procedure was successfully completed and the patients follow up, after a month, showed minimal limitation of the hand.

Conclusion: Advances in microsurgery allow multiple centers across the world to perform replantation of segments after a traumatic amputation. The success of procedure is based on the selection of candidates, the surgical team abilities and multidisciplinary management with better functional results than the use of prosthesis.
Volume 8 Issue 3 - 2018

Marcos Irving Salazar,' Jose Luis Monroy

Cedillo,' Luis Angel Medina Andrade, ${ }^{2}$ Ignacio

del Carmen Ortega,' Arianne Itzel del Rocio

Lupian Angulo,' Melissa Lomelí Canedo,' Jesus Ignacio Mazadiegio Cid, ${ }^{4}$ Paloma

Rosalva Albavera Gutierrez, ${ }^{2}$ Maria Fernanda

Aguilar Rodriguez, ${ }^{3}$ Yazmin Frias Lucio ${ }^{4}$

'General Surgery Resident, Hospital Central Norte de Petróleos Mexicanos, Mexico

${ }^{2}$ General Surgery Service, Hospital General de Zona IA

Rodolfo Antonio de Mucha Macias, Mexico

${ }^{3}$ Medical Student, Universidad Autónoma de Guadalajara, Mexico ${ }^{4}$ Occupational Medicine Service, Hospital General de zona \#32, Mexico

Correspondence: Luis Angel Medina Andrade, General

Surgery Service, Hospital General de Zona IA Rodolfo Antonio de Mucha Macias, IMSS, Mexico,

Email buismedina_5@hotmail.com

Received: June 04,2018 | Published: June 14, 2018

\section{Introduction}

Nowadays, in the microsurgery field, the surgeon has access to small vessels, nerves and lymphatics with the objective to decrease the limitation of the amputed extremity and maintain the functional status of the patient, improving life quality.

In 1871, Huerter, described the epineural suture and introduce the importance of free tension anastomosis.Years after, Alexis Carrell, in 1899, introduced the first vessel anastomos is technique by triangulation and thevein graft inter position to substitute damaged vascular tissue; these achievements got him the 1912 Nobel Price. ${ }^{1,2}$ In 1922, Holmgren introduced the binocular microscope Zeiss for medical use but it was until 1960 that Jacobsen and Suarez improved the outcome in small vessel anastomosis with new surgical equipment and microsurgical techniques. Based on this, in 1964, Smith, Michon and Marse established the bases of nervous microsurgery. ${ }^{1,3}$

Replantation is defined as the surgical procedure to reattach an amputated extremity with restoration of the neurovascular and musculoskeletal function and esthetic. ${ }^{3}$

\section{Case report}

A 32 year-old right-handed male patient without comorbilities is referred to our center with a traumatic amputation of the left hand within 6 hours after work accident with a wood saw.The initial management of the patient, consisted in mechanical lavageand closure of the wound achieving hemostasis, followed by compression bandage. The amputated hand is stored and transported in ice.
During the physical examination, the patient was awake with a Glasgow Coma Scale of 15 points, with vital sings within physiologic parameters, referring pain. The site of injury, was localized on the flexor zone II of the left hand, with adequate color and edges facing without any active bleeding. The left thumb was integrate without limitation in the range of movements, just a superficial lesion on the pulp(Figure 1a) (Figure 1b). The amputated segment, at the volar face, presented the injury at the level of metacarpophalangeal joint from the second to the fifth finger with regular cutaneous edges, the flexormuscles tendons with well-defined edges within the tendon sheaths (Figure 2)(Figure 3). From the dorsal view, the injury also showed regular cutaneous edges with extensor muscles tendons within the tendon sheaths.

The second finger presentedan irregular injury at the proximal interphalangeal joint with apparent fracture and other lesion at the distal phalange compromising the nail complex. The third finger showedan irregular transversal fracture at the medial phalanx level and the fourth finger presented an exposed and displaced fracture of the distal phalanx (Figure 4).

The patient is a candidate for replantation of the segment because of the time from the injury to medical attention, the age and no comorbilities, also the amputation of multiple fingers across the palm of the hand. The risk and benefits are explained to the patient and family, and accepted. 


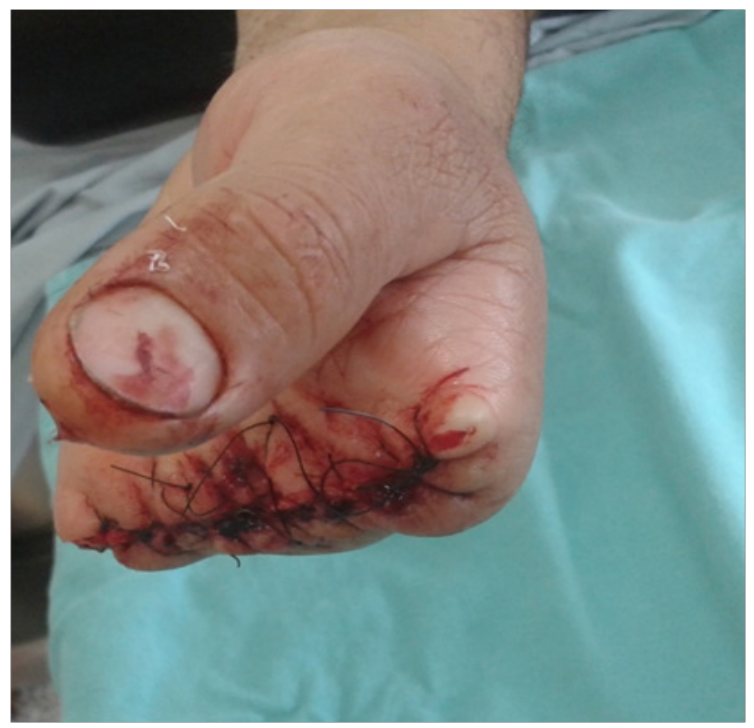

Figure I a The left hand with amputation in the flexor zone II.

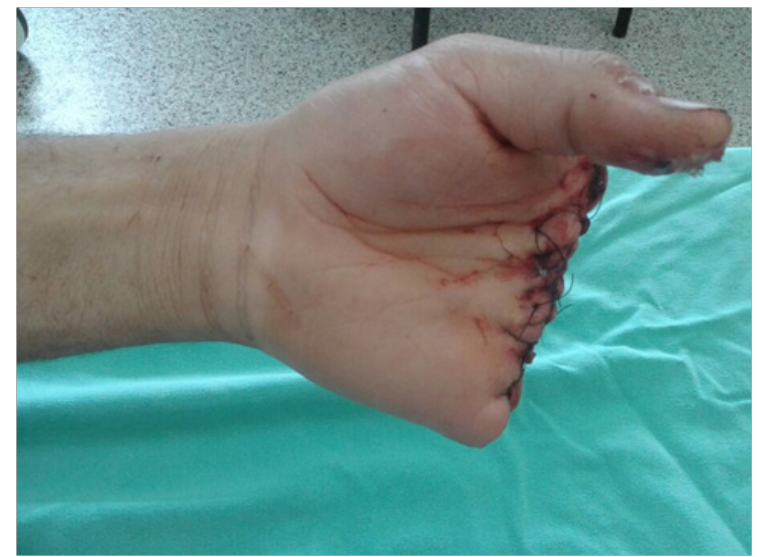

Figure I b The left hand with amputation in the flexor zone II.

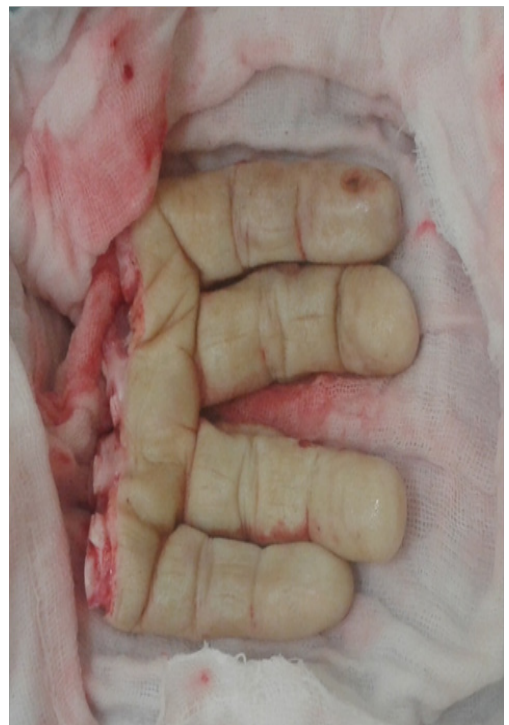

Figure 2 Volar side of the amputated segment.

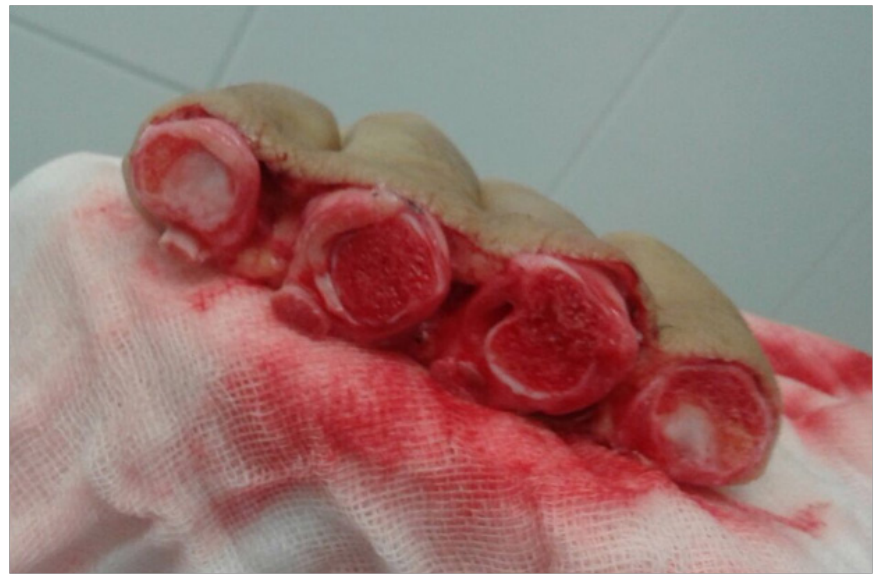

Figure 3 Anterior view of the amputated segment.

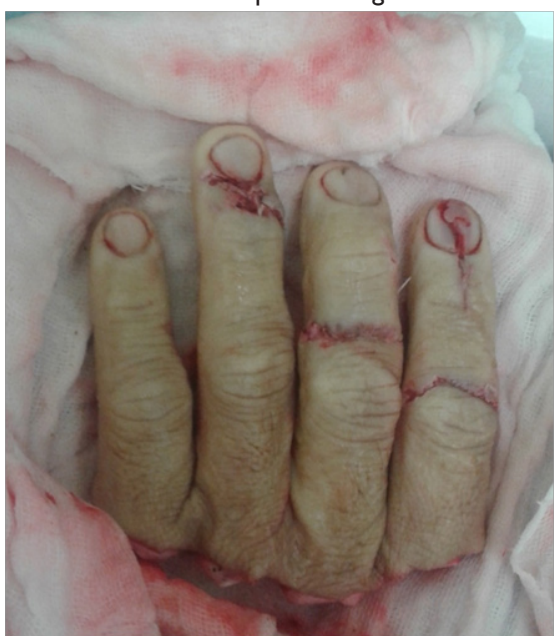

Figure 4 Dorsal view of the amputated segment.

\section{Surgical intervention}

Under general anesthesia, the procedure begins with debridement of edges and mechanical lavage with Ringer slactate and iodopovidone of the amputated segment until bleeding through the wound is observed.

During the first surgical intervention, the metacarpophalangeal fractures were stabilized with the closure of the lumbrical and interosseous muscles with an absorbable suture (Vycril 3/0) without the need of osteosynthesis material. The repair of the tendon was with modified. Kessler stitches with a non-absorbable suture (Prolene 4/0), including the digitorum extensor, indicis, digitiminimi and exors like digitorum superficialis and profundus.

The second intervention was realized with microsurgical exploration (10x) where the two transmetacarpal dorsal veinspresented with well-defined edges; the anastomosis of the vessels was performed with nylon $8 / 0$. On the volar side, the palmar-digital arteries also presented regular edges, the anastomosis was performed with nylon $8 / 0$. No nervous repairs were made at this point. Skin is closed with nylon 3/0 simple stitches, splinting and bandaging.

The postoperative period is coursed in the Intensive Care Unit with 
pulse oximetry check up every 2 hours, for the first two days, reporting above $96 \%$. The color and temperature were evaluated and compared with the contralateral hand. Analgesia was provided with Opiods (Tramadol 200mg every 24hours) and NSAIDS (ketorolaco 30mg every 8hours); Broad-spectrum antibiotics and thromboprophylaxis with low molecular weight heparin were administered too.

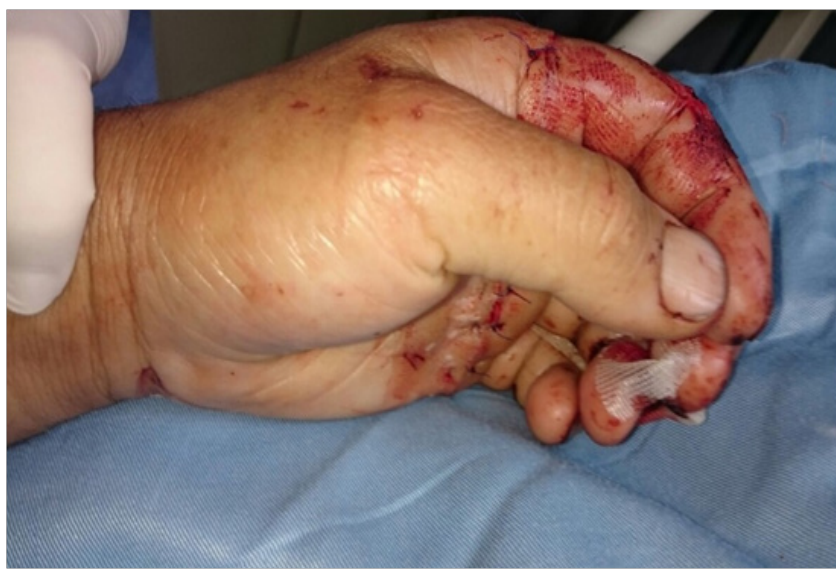

Figure 5 Reimplanted hand 24 hours after surgery.

\section{Discussion}

The first registry of a successful reimplant was in 1814 when William Belfour publish a digital reimplantation in the Edinburgh Medical and Surgical Journal, where he reimplanted successfully his son's fingers, although those reimplantations were performed without vascular anastomosis, and for this reason is suspected that they survive as composed grafts. In 1964 Douglas and Foster introduced the theory of spontaneous recanalization that can occur in composed grafts. ${ }^{1}$

However, it was until 1962 when S. Ronald Malt and Mckhann in Boston, accomplished the first replantation of an arm amputated above the shoulder, in a 12 year-old boy that had traumatic amputation in a train accident, It is the first successful replantation registered. ${ }^{1,2}$

In 1963, Kleinert et al. performed the first anastomosis of a digital artery for the revascularization of partially amputated thumb, and in 1965 Komatsu and Tamai, in Japan, completed the first complete thumb replantation, setting a standard in the functional replantation surgery. ${ }^{1,2}$ For the last 20 years, successful replantation cases have been published because of the development of surgical microscopes, ultrafine surgical sutures and grafts, also the surgical skills of the surgeons involved. ${ }^{3}$

In the setting of a traumatic amputation, them echanism is very important in relation with the tissue damage, determining if the patient is a candidate or not to a replantation procedure. For this reason, crushing injury, which are the most frequent injuries, represent a challenge because of need for tissue debridement and the shortening of structures until healthy tissue is appropriate for a tension-free anastomosis, diminishing the percentage of viability.,

It is reported, specific criteria for the selection of patients, candidates for a replantation, considering factors like comorbilities, the probability of survival of the affected segment and the function of the replanted segment could be the same or better than the one achieved with a prosthesis. ${ }^{6}$
The patient courses a successful postoperative period andis discharged two weeks after surgery. Four weeks after discharge he began rehabilitation 3 times per week. The patient will require another procedure for interphalangeal fracture fixation by osteosynthesis (Figure 5) (Figure 6).

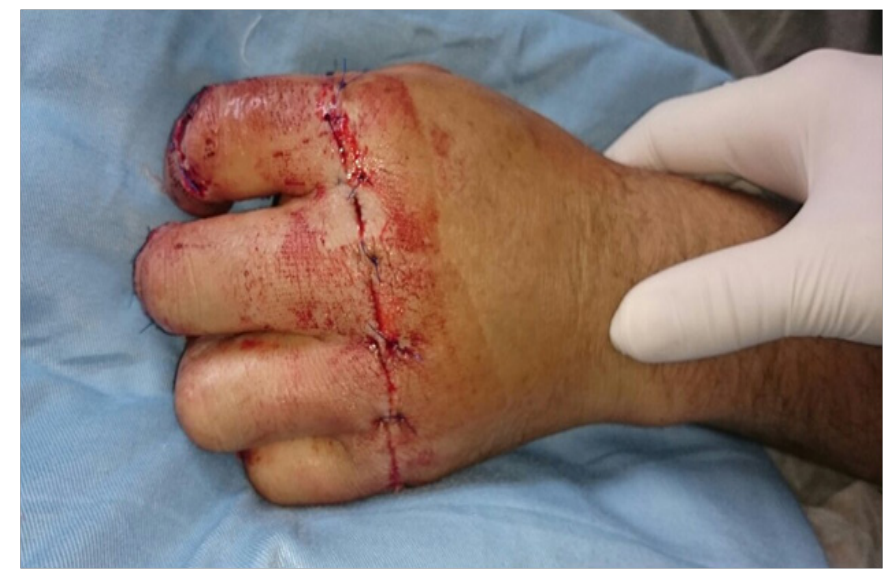

Figure 6 Reimplanted hand 7 days after surgery.

The success of the viability of a tissue cannot be compared to functional success of the replanted segment, and the indications mentioned are recommendations of experts and cannot be interpreted as absolute either ${ }^{6}$ (Table 1). Generally, a successful replantation of an amputation at the level of palm, wrist or distal end of the forearm provide a better function than the one achieved with a prosthesis, by the grip and release provided by the extrinsic muscles of the fingers. ${ }^{6}$

The preservation of the amputated segment is vital to the success of replantation. In the literature, its recommended the replantation before 12 hours of warm ischemia for the fingers and 6 hours for a major replantation of the upper extremity, and in case of cold ischemia, it must be maximum of 24 hours and 12 hours respectively. ${ }^{6}$ The amputated segment must be submerged in saline solution or gauze soaked from the same solution, put in a plastic bag and submerged in a solution with ice at $4^{\circ} \mathrm{C}$, freezing or direct contact with ice must be avoided to prevent more tissue damage., ${ }^{4,6}$

In general, the patient has big expectations on the outcome of replantation, for this reason, the surgeon must explain the possibilities of success, viability, possible functional results, surgical time, days of hospitalization and rehabilitation therapy must accomplish to improve the results. ${ }^{3,7}$ More than 60 years after the first reported replantation, there have been multiple successful out comesincluding hand and fingers. The main factors for successful replantation are age, concomitant lesions, injury mechanism and time of ischemia. ${ }^{1,4}$

A successful replantation require a multidisciplinary team with experience the area and microsurgical equipment for reconstructive surgery. It is important to mention that the tissue viability is not equal to success, determined by the sensitive and motor function preserved in the implanted segment. ${ }^{8,910}$ In previous studies it has been demonstrated that younger patients with guillotine type amputations have a higher rate of success after reimplantation, reaching a $94 \%$ of success. In cases of avulsion, they have a bad prognosis. In the case of our patient with an amputation at the palm level and cold ischemia of 
6 hours, it was justified to practice the replantation with an excellent final result. ${ }^{2,9,11}$

\section{Conclusion}

Although there is high rate of success in the replantation procedures with microsurgery, in developing countries because of expensive cost of procedures, prolonged incapacity period and rehabilitation, it is not accesible for the population, however, it must be considered in order to restore the function of the extremity., $, 9,12$ The experts recommendation cannot be absolute, and each patient must be individualized in their management, considering the final functional results that could be obtained. , $13^{13}$

\section{Acknowledgments}

None.

\section{Conflict of interest}

Author declares no conflict of interests.

\section{References}

1. Kocher MS. History of replantation: from miracle to microsurgery. World J Surg.1995;19(3):462-7.

2. Friedrich JB, Poppler LH, Mack CD, et al. Epidemiology of upper extremity replantation surgery in the United States. J Hand Surg Am. 2011;36(11):1835-40.
3. Neumeister MW, Brown RE. Mutilating hand injuries: principles and management. Hand Clin. 2003;19(1):1-15,

4. Jaeger SH, Tsai TM, Kleinert HE. Upper extremity replantation in children. Orthop Clin North Am. 1981;12(4):897-907.

5. Sugun TS, Ozaksar K, Ada S, et al. [Long-term results of major upper extremity replantations]. Acta OrthopTraumatol Turc. 2009;43(3):20613.

6. Chung KC, Alderman AK. Replantation of the upper extremity: indications and outcomes. J Am Soc Surg Hand. 2002;2(2):78-94.

7. Manord JD, Garrard CL, Kline DG, et al. Management of severe proximal vascular and neural injury of the upper extremity. $J$ Vasc Surg. 1998;27(1):43-7.

8. PatelAA, Blount AL, Owens PW, et al. Functional outcomes of replantation following radiocarpal amputation. J Hand Surg Am. 2015;40(2):266-70.

9. Sabapathy SR, Venkatramani H, Bharathi RR, et al. Replantation surgery. J Hand Surg Am. 2011;36(6):1104-10.

10. Reichl H, Schütz T, Gabl M, et al. [Hand replantation: differences in functional outcome considering patient age and socio-medical aspects]. Handchir Mikrochir Plast Chir. 2013;45(6):344-9.

11. Ronald AM, Charles M. Replantation of severed arms. JAMA. 1964;189:716-722

12. Hierner R, Berger A, Brenner P. [Considerations on the management of subtotal and total macro-amputation of the upper extremity]. Unfallchirurg. 1998;101(3):184-192. 